\title{
Symposium: Neuroergonomics, technology, and cognition
}

\author{
Raja Parasuraman ${ }^{\mathrm{a}, *}$, Carryl L. Baldwin ${ }^{\mathrm{a}}$, Benjamin Knott ${ }^{\mathrm{b}}$, Joel S. Warm ${ }^{\mathrm{b}}$, Victor Finomore ${ }^{\mathrm{b}}$, Deborah \\ Boehm-Davis $^{\mathrm{a}}$, and Scott M. Galster ${ }^{\mathrm{b}}$ \\ ${ }^{a}$ Department of Psychology, George Mason University, 4400 University Drive, Fairfax, VA 22030, USA \\ ${ }^{\mathrm{b}}$ Air Force Research Laboratory, 711 Human Performance Wing, Wright-Patterson AFB OH 45433, USA
}

\begin{abstract}
This symposium describes collaborative research on neuroergonomics, technology, and cognition being conducted at George Mason University and the US Air Force Research Laboratory (AFRL) as part of the Center of Excellence in Neuroergonomics, Technology, and Cognition (CENTEC). Six presentations describe the latest developments in neuroergonomics research conducted by CENTEC scientists. The individual papers cover studies of: (1) adaptive learning systems; (2) neurobehavioral synchronicity during team performance; (3) genetics and individual differences in decision making; (4) vigilance and mindlessness; (5) interruptions and multi-tasking; and (6) development of a simulation capability that integrates measures across these domains and levels of analysis.
\end{abstract}

Keywords: Neuroergonomics, technology, cognition.

\section{Introduction}

Ergonomics seeks to design systems and work environments that are compatible with human capabilities and limitations, both physical and mental, and to select and train human operators in such systems. Neuroergonomics has the same objective, but proposes that examining human brain function, in addition to behavior, can help in achieving that goal. The central premise of neuroergonomics is that research and practice in human factors and ergonomics can be enriched by consideration of theories and results from neuroscience.

Some decades ago such a claim would have been considered far-fetched because our knowledge of human brain function was limited and restricted to only the simplest aspects of human behavior. But the phenomenal growth of human cognitive neuroscience has lead to an increasing number of studies that have enhanced understanding of human behavior and suggested ways of improving human performance in such work domains as aviation, ground transportation, education, security, and the military, as well as in many everyday settings.
The emerging, inter-disciplinary field of neuroergonomics has experienced rapid growth in recent years, both in the US as well as in Europe. A special issue on the topic first appeared in an ergonomics journal [1] followed by a book [2], a special issue in a neuroscience journal [3] and by a growing number of journal articles and papers in conferences. The field has also had international impact, as evidenced by the establishment by ergonomists of neuroergonomic research units in countries such as Poland [4], Germany [5], and elsewhere. Much of this research activity has been fueled by the emergence of substantial research and training grant funding provided by different agencies. One such effort, involving research, training, and collaborative work is the Center of Excellence in Neuroergonomics, Technology, and Cognition (CENTEC), established in 2010 at George Mason University (GMU) under the sponsorship of the US Air Force Research Laboratory (AFRL).

This symposium features scientists who are part of CENTEC at GMU and at AFRL. The goal of the symposium is to provide a focused survey of the latest developments in neuroergonomics and to highlight the collaborative research efforts between GMU

\footnotetext{
${ }^{*}$ Corresponding author. Email: rparasur@gmu.edu
} 
and AFRL scientists in this emerging field. The individual papers cover several topics, including studies of: (1) adaptive learning systems; (2) neurobehavioral synchronicity during team performance; (3) genetics and individual differences in decision making (4) interruptions and multi-tasking; (5) vigilance and mental workload; and (6) development of a simulation capability that integrates measures across these domains and levels of analysis.

The papers will illustrate how the use of behavioral and neuroimaging (EEG, MRI, genetic) measures can enhance understanding of each of these aspects of ergonomic issues. Overall, the objective of the symposium is to provide IEA attendees with a better understanding of the scope of neuroergonomics, its application to the solution to ergonomic problems, and the prospects for the future.

\section{Neuro-adaptive learning systems (Carryl L. Baldwin)}

Individualized adaptive training platforms can improve the efficiency and utilization of limited educational resources. Information acquisition and mastery of new skills is an on-going challenge in educational, employment, and military settings. In an ideal training environment, material should be presented in a format and at a rate that keeps the learner engaged and challenged without leading to boredom or cognitive overload. Performance metrics alone may fail to adequately indicate the underlying emotionalcognitive state of the learner and large individual differences in prior knowledge, working memory capacity, and emotional-cognitive learning styles may render static approaches inefficient.

This presentation describes recent work involving using neurophysiological metrics to drive artificial neural networks (ANNs) for real-time learner emotional-cognitive state classification. Primary metrics included pupilometry and EEG metrics that are well established in the extent literature as well as several innovative new approaches. The training environment presents several unique challenges to operator state classification and our success to date towards meeting these challenges will be discussed. Unlike more traditional paradigms aimed at operator functional state classification, the trainee is by definition performing novel tasks or levels of tasks. Therefore classification procedures must be able to predict performance on novel tasks.

In a recent series of experiments we have examined the classification accuracy of ANNs trained on specific working memory tasks for novel working memory tasks (cross-task classifications) in a series of experiment. Lessons learned and progress towards developing adaptive training platforms will be discussed.

\section{Neurobehavioral synchronicity as a correlate of team performance (Benjamin Knott)}

Effective teamwork is essential to many military, industrial, medical, and public service work environments. Researchers of team performance are therefore frequently interested in identifying the behavioral, perceptual, and cognitive processes that contribute to team performance and methods for measuring and supporting teamwork in these domains. For example, researchers have found that team communication is a moderate predictor of team performance and team cohesion [6]. Similarly, research suggests that team cohesion is a moderate predictor of team performance and that this relationship is stronger for sport and military teams compared to those that might be composed artificially in laboratory experiments.

More recently, several investigators have attempted to examine factors associated with peripheral and autonomic nervous system function that might underlie and contribute to team processes and performance [7-9]. For example, Henning et al. [7] observed a cross correlation, or synchronicity, in cardiac interbeat intervals (IBIs) in dyads performing a cooperative computer task. Moreover, greater IBI synchronicity was associated with better team performance on the task. Similarly, Shockley and colleagues [9] found that when teams worked cooperatively on solving a visual puzzle, their postural sway became synchronized, but that synchronicity did not occur when participants performed the same task independently in the presence of another individual. These and similar findings are significant because they demonstrate that certain physiological or neurobehavioral responses could be used in the future as metrics of team process or as predictors of team performance.

An experiment will be presented in which twoperson teams played a cooperative computer game, and team-paired IBIs and postural sway (anteriorposterior head motion) were analyzed using CrossSample Entropy (CSEn) as an indication of neurobehavioral synchronicity. In addition, the quantity of team verbal communication (number of words spoken) and a survey measure of team cohesion were assessed. Team performance was found to be associated with a decrease in IBI synchronicity, while an 
increase in team verbal communication was related to both an increase in postural sway synchronicity and team cohesion. The implications of this research will be discussed in terms of the notion that metrics of team synchronicity could serve as useful indices of team processes and performance in military task domains.

\section{Neurogenetics of individual differences in com- plex decision making (Raja Parasuraman)}

The timeliness and accuracy of decision making are dependent on two cognitive functions that can vary between individuals and that can also be trained: working memory and executive function. Both are dependent on the functioning of prefrontal cortex and its dopaminergic innervation [10]. This presentation therefore examines associations between working memory and decision making and genes that influence dopamine signaling in the brain, in particular the $-1021 \mathrm{C} / \mathrm{T}$ single nucleotide polymorphism (SNP) of the dopamine hydroxylase (DBH) gene. A group of 108 adults aged $18-45$ years was genotyped for the $-1021 \mathrm{C} / \mathrm{T}$ SNP and subdivided into three sub-groups based on the $\mathrm{CC}$, TC, and TT allele combinations. Participants performed a spatial working memory task and a simulated command and control task.

The working memory task involved maintaining a representation of up to three spatial locations over a period of $3 \mathrm{~s}$. Working memory accuracy for the TT sub-group was significantly greater than that for individuals with the TC and CC genotypes. The decision making task required participants to identify the most dangerous enemy target in a terrain view and engage it within $10 \mathrm{~s}$, either manually or with automation support ( $80 \%$ reliable). Accuracy of enemy engagement decisions did not differ as a function of DBH genotype. However, decision time was significantly lower in individuals with the TT genotype than for CC individuals, both when the task was performed manually and with the help of automation. Decision making performance was poorer on trials when the automation provided incorrect recommendations than when the automation was reliable. However, this cost of automation unreliability was reduced in the TT group compared to the TC and CC groups. The results indicate that the $-1021 \mathrm{C} / \mathrm{T} \mathrm{SNP}$ of the DBH gene is associated with individual variation in working memory capacity and with speeded decision making under time pressure. Both findings are consistent with a prominent role for prefrontallymodulated working memory processes in decision making. Implications for training decision making under time pressure are discussed.

\section{The SART task does not promote mindlessness in vigilance (Joel S. Warm and Victor Finomore)}

Vigilance or sustained attention tasks are critical elements in many work environments ranging from air-traffic control, airport and border security, industrial quality control, and the inspection of medical gauges during surgery. These tasks require observers to monitor displays for extended periods of time and detect the appearance of critical signals. In a traditional experimental format, the signals are embedded in a background of neutral or non-signal events and observers are required to make an overt response such as a button press to the critical signals and make no response to the more frequent neutral events. Thus, in the traditional format, vigilance tasks can be described as "go/no go" attentional assignments in which the frequency of "no-go" events outweighs that of "go" events.

Currently, there are two competing models to account for failure of signal detection in vigilance tasks. One of these is the resource model in which the need to make continuous signal/noise discriminations is held to deplete observers' information-processing assets leading to missed signals [11-12]. An alternative view is the mindlessness model in which the repetitive nature of vigilance tasks is considered to lead to a lack of attentional focus and thence to failures of signal detection. Proponents of this model have developed a new version of the vigilance task known as the Sustained Attention to Response Task (SART), which is designed to promote mindlessness in vigilance by inverting the "go/no-go" ratio [13]. With this task, observers are asked to respond to the more frequent neutral events and to withhold responding in the presence of the less frequent critical signals.

The present study was designed to assess the validity of the claim that the SART is an engine to promote mindlessness in vigilance. Toward that end, the SART and the traditional vigilance formats were compared on measures of perceived mental workload assessed via the Multiple Resource Questionnaire (MRQ), active visual scanning of the monitored display indexed via an eye scanning measure, the nearest neighbor index, and cerebral blood flow velocity measured by means of Transcranial Doppler Sonography. It was anticipated that if the SART does indeed promote mindlessness, its associated workload, visual scanning, and cerebral blood flow velocity 
(CBFV) should be less than that of a traditional format (TVF) task. None of these expectations was borne out. The SART and the TVF had identical workload profiles on the MRQ which were at the upper level of the scale, scanning was equally active in the two types of vigilance tasks and more vigorous than in a passive control group who monitored the vigilance display without a work imperative, and CBFV declined over time in a similar manner in both task formats while it remained stable over time in the passive control group. The results of this study challenge the validity of the proposition that detection failures in the SART emanate from a withdrawal of attentional effort. In so doing they challenge the viability of the mindlessness model of vigilance that draws its major support from research with the SART.

\section{Interruptions and multitasking (Deborah A. Boehm-Davis)}

Few of us have the luxury of working without interruptions of one sort or another. In most workplaces, jobs arrive independent of jobs already in the queue, workers need to consult with each other to pursue parallel work streams, and jobs are suspended when parts or information needed are not readily available. In short, interruption is the norm in almost all work environments, including aviation, ground transportation, medicine, education, security, and the military. In concert with this, people are more likely to engage in multitasking now than they have been in the past. New technologies allow users to have multiple applications open and they often shift focus from application to application rather than working on tasks in a strictly serial fashion. As a result, it is more important than ever to understand the processes by which people engage in, disengage from, and return to tasks. This presentation will present two theoretical models, one focusing on what is known about interruptions and one focusing on what is known about multitasking. Gaps in the literature will be identified and some initial research in which we are engaged to explore these gaps will be described.

\section{HUMAN: Human Universal Measurement and Assessment Network (Scott M. Galster)}

Throughout the 1990's, designated the "Decade of the Brain", and in recent years, the technologies and methods of cognitive science, neuroscience, and psychophysiology have matured such that they are poised to move from the lab into the field.
Neuroscience oriented technologies include invasive and non-invasive neuroimaging, computational modeling, psychophysiological assessment, and advanced signal processing for real time identification of operator states. Leading researchers employ the term neuroergonomics to describe their study of the brain and its behavior during work $[1,2]$.

Air, Space, and Cyber battlefield operations are becoming increasingly automated and complex due to rapid technology changes and changing threats. The Human Universal Measurement and Assessment Network (HUMAN) Program is most interested in findings and aspects of technologies that can be demonstrated in one domain but then generalized across other domains. For example, commanders' information requirements have resulted in concepts of operations (CONOPS) that include multiple remotely piloted aircraft (RPA) with multiple miniaturized advanced sensors. These CONOPS feature teams of operators that must simultaneously control multiple heterogeneous RPA with multiple sensors. CONOPS such as these necessitate assessment of operator and team cognitive workload, as well as individually and system validated neuroergonomic technologies. Once air domain specific team cognitive workload management technologies and design approaches are validated, cyber and space domain commanders having similar team issues will likely benefit.

Efforts employed to establish the HUMAN laboratory and the integration of the current technologies and methodologies to sense, assess, and augment individual and team performance will be described in this presentation.

\section{Acknowledgments}

Supported by AFOSR/AFRL grant FA9550-10-10385 and the Center of Excellence in Neuroergonomics, Technology, and Cognition (CENTEC).

\section{References}

[1] Parasuraman, R. (Ed.). (2003). Neuroergonomics. Special issues of Theoretical Issues in Ergonomics Science. 4(1) and 4 (2).

[2] Parasuraman, R., \& Rizzo, M. (2007). Neuroergonomics: The brain at work. New York: Oxford University Press. 
[3] Parasuraman, R., Christensen, J., \& Grafton, S. (Eds.) (2011) Neuroergonomics: The human brain in action and at work. NeuroImage, 58. doi:10.1016/j.neuroimage.2011.08.011,

[4] Fafrowicz, M., \& Marek, T. (2007). Quo vadis, neuroergonomics? Ergonomics, 50, 1941-1949.

[5] Stork, S., Stobel, C., Muller, H. J., Wiesbeck, M., Zah, M .F., $\&$ Schubo, A. (2007). A neuroergonomic approach for the investigation of cognitive processes in interactive assembly environments. In Proceedings of the 16th IEEE International Symposium on Robot and Human Interactive Communication, pp. 750-755.

[6] Barrick, M.R., Stewart, G.L., Neubert, M.J., \& Mount, M.K. (1998). Relating member ability and personality to work-team processes and team effectiveness. Journal of Applied Psychology, 83, 377-391.

[7] Henning, R.A., Boucsein, W., \& Gil, M.C. (2001). Socialphysiological compliance as a determinant of team performance. International Journal of Psychophysiology, 40, 221232.

[8] Shockley, K., Baker, A.A., Richardson, M.J., \& Fowler, C.A. (2007). Articulatory constraints on interpersonal postural coordination. Journal of Experimental Psychology: Human Perception and Performance, 33, 201-208.

[9] Shockley, K., Santana, M.V., \& Fowler, C.A. (2003). Mutual interpersonal postural constraints are involved in cooperative conversation. Journal of Experimental Psychology: Human Perception and Performance, 29, 326-332.

[10] Parasuraman, R., \& Jiang, Y. (2011). Individual differences in cognition, affect, and performance: Behavioral, neuroimaging, and molecular genetic approaches. NeuroImage. doi:10.1016/j.neuroimage.2011.04.040

[11] Parasuraman, R. (1979). Memory load and event rate control sensitivity decrements in sustained attention. Science, 205, 924-927.

[12] Warm, J. S., Parasuraman, R., \& Matthews, G. (2008). Vigilance requires hard mental work and is stressful. Human Factors, 50, 433-441.

[13] Robertson, I. H., Manly, T., Andrade, J., Baddeley, B. T., \& Yiend, J. (1997). “Oops!" Performance correlates of everyday attentional failures in traumatic brain injured and normal subjects. Neuropsychologia, 35, 747-758. 